\title{
TLR3 agonist and CD40-targeting vaccination induces immune responses and reduces HIV-1 reservoirs
}

\author{
Liang Cheng, ${ }^{1,2}$ Qi Wang, ${ }^{1}$ Guangming Li, ${ }^{1}$ Riddhima Banga, ${ }^{3,4}$ Jianping Ma, ${ }^{1}$ Haisheng Yu, ${ }^{1}$ Fumihiko Yasui, ${ }^{1}$ Zheng Zhang, \\ Giuseppe Pantaleo, ${ }^{3,4}$ Matthieu Perreau, ${ }^{3,4}$ Sandra Zurawski, ${ }^{6,7}$ Gerard Zurawski, ${ }^{6,7}$ Yves Levy, ${ }^{6,8}$ and Lishan Su ${ }^{1,2}$ \\ 'Lineberger Comprehensive Cancer Center, and ${ }^{2}$ Department of Microbiology and Immunology, University of North Carolina at Chapel Hill, Chapel Hill, North Carolina, USA. ${ }^{3}$ Service of Immunology and \\ Allergy and ${ }^{4}$ Swiss Vaccine Research Institute, Lausanne University Hospital, University of Lausanne, Lausanne, Switzerland. ${ }^{5}$ Research Center for Clinical \& Translational Medicine, Beijing 302 Hospital, \\ Beijing, China. ${ }^{6}$ Vaccine Research Institute, Université Paris-Est, Faculté de Médecine, INSERM U955, Créteil, France. ${ }^{7}$ Baylor Institute for Immunology Research and INSERM U955, Dallas, Texas, USA. \\ ${ }^{8}$ Assistance Publique-Hôpitaux de Paris, Groupe Henri-Mondor Albert-Chenevier, Service d'Immunologie Clinique, Créteil, France.
}

Activation of HIV-1 reservoirs and induction of anti-HIV-1 T cells are critical to control HIV-1 rebound after combined antiretroviral therapy (cART). Here we evaluated in humanized mice (hu-mice) with persistent HIV-1 infection the therapeutic effect of TLR3 agonist and a CD40-targeting HIV-1 vaccine, which consists of a string of 5 highly conserved CD4 $4^{+}$and CD8 ${ }^{+}$ T cell epitope-rich regions of HIV-1 Gag, Nef, and Pol fused to the C-terminus of a recombinant anti-human CD40 antibody ( $\alpha$ CD40.HIV5pep). We show that $\alpha$ CD40.HIV5pep vaccination coadministered with poly(I:C) adjuvant induced HIV-1-specific human $\mathrm{CD}^{+}$and $\mathrm{CD4}^{+} \mathrm{T}$ cell responses in hu-mice. Interestingly, poly(I:C) treatment also reactivated HIV-1 reservoirs. When administrated in therapeutic settings in HIV-1-infected hu-mice under effective CART, $\alpha$ CD40.HIV5pep with poly(I:C) vaccination induced HIV-1-specific CD8 ${ }^{+}$T cells and reduced the level of cell-associated HIV-1 DNA (or HIV-1 reservoirs) in lymphoid tissues. Most strikingly, the vaccination significantly delayed HIV-1 rebound after cART cessation. In summary, the $\alpha$ CD40.HIV5pep with poly(I:C) vaccination approach both activates replication of HIV-1 reservoirs and enhances the antiHIV-1 T cell response, leading to a reduced level of cell-associated HIV-1 DNA or reservoirs. Our proof-of-concept study has significant implication for the development of CD40-targeting HIV-1 vaccine to enhance anti-HIV-1 immunity and reduce HIV-1 reservoirs in patients with suppressive CART.

\section{Introduction}

Combined antiretroviral therapy (cART) suppresses viral replication and improves survival and quality of life for those HIV-1-infected patients who can both access and tolerate cART. However, cART is not curative and must be continued for life (1-3). The HIV-1 reservoir persists indefinitely under suppressive cART, resulting in viral rebound in all HIV-1-infected individuals when cART is discontinued. In addition, cART does not fully restore immune function and lifelong treatment is associated with substantial side effects and non-AIDS related "end-organ diseases"(4). Therefore, there is a great need for the development of novel therapies to control or eliminate the persistent HIV-1 reservoir and thus reduce the need for lifelong cART.

During natural HIV-1 infection, a limited number of individuals (termed elite controllers or long-term nonprogressors) remain persistently infected for decades without marked depletion of $\mathrm{CD}^{+} \mathrm{T}$ cells $(5-7)$. These patients exhibit strong HIV-1-specific polyclonal memory $\mathrm{CD}^{+} \mathrm{T}$ cell activity and maintain a highly polyfunctional cytotoxic T lymphocyte (CTL) response (8-10). The robust HIV-1-specific immune response observed in a large majority of these patients has been proposed to explain this natu-

Conflict of interest: $\mathrm{GZ}, \mathrm{SZ}$, and $\mathrm{YL}$ are named inventors on CD40-targeting vaccine patents and patent filings held jointly by INSERM and the Baylor Research Institute. Submitted: December 4, 2017; Accepted: July 9, 2018.

Reference information: J Clin Invest. 2018;128(10):4387-4396.

https://doi.org/10.1172/JCI99005. ral functional cure, raising the possibility that therapeutic immunization in infected patients whose viral replication is suppressed by cART might result in similar control of viral replication after cART discontinuation (11-13).

Therapeutic immunization is intended to enhance the immune responses against HIV-1 by vaccination with a suitable immunogen. One way to enhance the immunogenicity of proteins is to increase their uptake by dendritic cells (DCs), which are specialized antigen-presenting cells (APCs) (14-16). Targeting DCs by fusing antigens to monoclonal antibodies (mAbs) directed against internalizing cell-surface receptors can substantially enhance protein immunogenicity (17-19). We and others have demonstrated that targeting HIV-1 Env gp140 or Gag p24 to various DC receptors including DEC-205, LOX-1, Langerin, DCIR, and CD40 induced potentially protective humoral and cellular immunity in both priming and viral vector boost settings (20-23). Recently, we tested in both nonhuman primates (NHPs) and in humans an epitope-based vaccine composed of 5 HIV-1 peptides which contain multiple and highly conserved $\mathrm{T}$ cell epitopes from HIV-1 Gag, Pol, and Nef that induce HIV-1-specific CD4 $4^{+}$and $\mathrm{CD}^{+} \mathrm{T}$ cell responses (24-29). Moreover, we have shown that administration of these T cell epitopes as HIV-1 lipopeptides either combined with a recombinant virus (CanaryPox) or as a component of an ex vivo DC therapeutic vaccine strategy, contributed to the control of viral replication after cART interruption $(27,30,31)$.

CD40 is a potent activating receptor expressed by a range of APCs, including DCs (32). Thus, targeting CD40 offers the poten- 
A

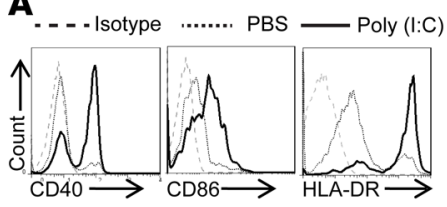

C

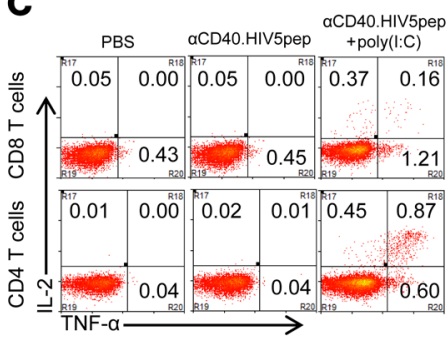

$\mathbf{B}$
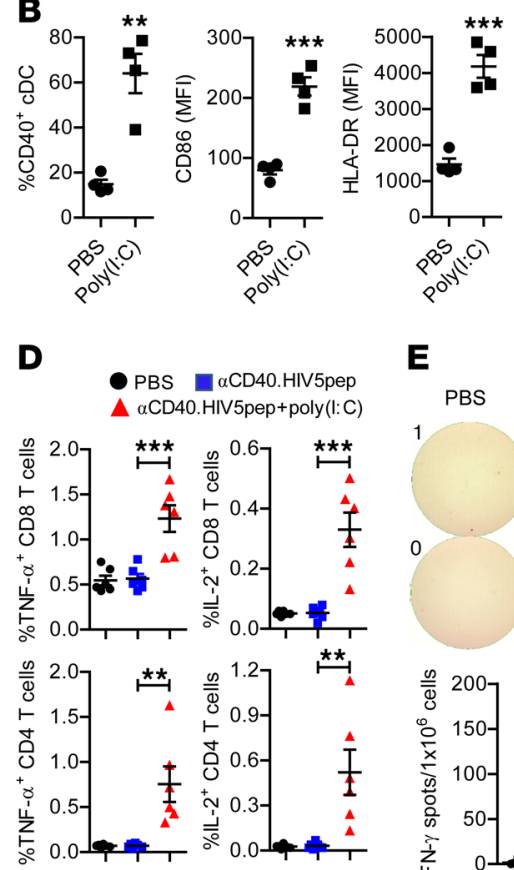

Figure 1. Poly(l:C) enhances $\alpha C D 40$.HIV5pep vaccination to induce both human $\mathrm{CD}^{+}$and $\mathrm{CD}^{+} \mathrm{T}$ cell responses in vivo. (A and B) Hu-mice were treated with poly(l:C) $(50 \mu \mathrm{g} /$ mouse, i.p.) or PBS as control. Leukocytes from spleen were isolated for flow cytometry analysis 24 hours after treatment. Representative histogram (A) and summary data (B) show the expression of CD40, CD86, and HLA-DR on mDCs (hCD45 ${ }^{+} \mathrm{CD3}^{-}$ CD19-CD14-CD11C $C^{+}$after treatment. (B) Representative data from 4 hu-mice per group with mean values \pm SEM of 3 independent experiments. ${ }^{* *} P<0.01,{ }^{* *} P<0.001$ by unpaired, 2 -tailed Student's $t$ test. (C-E) Hu-mice were vaccinated with $\alpha$ CD40.HIV5pep with or without poly(I:C) and boosted at week 3. Mice were euthanized 10 days after the boost vaccination. Splenocytes from mice were stimulated ex vivo with the corresponding 5 specific HIV-1 long peptides plus $\alpha$ CD28 mAb. (C and D) IL-2 and TNF- $\alpha$ expression by CD8 ${ }^{+}$ and $\mathrm{CD} 4^{+} \mathrm{T}$ cells were detected by intracellular staining. Representative plots (C) and summarized data (D) show percentages of IL-2- and TNF- $\alpha$-producing $C D 8^{+} T$ cells $C D 4^{+}$T cells. (E) IFN- $\gamma$ production was detected by ELISpot assay. ( $\boldsymbol{D}$ and $\mathbf{E}$ ) Combined data from 2 independent experiments with mean values \pm SEM (PBS, $n=6 ; \alpha C D 40$. HIV5pep, $n=6, \alpha$ CD40. HIV5pep + poly(I:C), $n=6)$. ${ }^{*} P<0.05,{ }^{* *} P<0.01,{ }^{* *} P<0.001$ by 1-way ANOVA and Bonferroni's post hoc test.

tial advantage of inducing DC maturation and delivery of antigen to $\mathrm{CD} 40$-induced antigen-specific humoral and cellular immune response $(33,34)$ and protection against tumor $(35)$. In addition, CD40 targeting has shown superiority for evoking antigenspecific $\mathrm{CD} 8^{+} \mathrm{T}$ cell responses in vitro over targeting other receptors, including DEC-205, DCIR, and LOX-1 (36-38). We have fused the above-mentioned 5 HIV-1 peptides to the heavy chain C-terminus of a recombinant anti-human CD40 antibody (called herein $\alpha$ CD 40.HIV5pep) (39). The $\alpha$ CD 40.HIV5pep can effectively expand HIV-1 antigen-specific multifunctional helper $\mathrm{CD} 4^{+}$and cytotoxic CD8 ${ }^{+} \mathrm{T}$ cells in HIV-1-infected patient PBMC or autologous DC/T cell cocultures (39). The expanded polyfunctional cytotoxic $\mathrm{CD}^{+} \mathrm{T}$ cells can control HIV-1 replication in vitro (39). In addition, Toll-like receptor ligand (TLR-L) adjuvants that can activate and elevate CD40 expression on human APCs (40) may synergistically enhance the efficacy of the CD40-targeting vaccine. We have showed previously that poly(I:C) was superior to other TLR agonist adjuvants (CpG-A, CpG-B, CpG-C, R848, R837, MPLA) for inducing human IL-12 production and activating human mDCs both in vitro and in vivo in humanized mice (hu-mice) (40). Importantly, we believe we have also proven that poly $(\mathrm{I}: \mathrm{C})$ is more efficacious
E

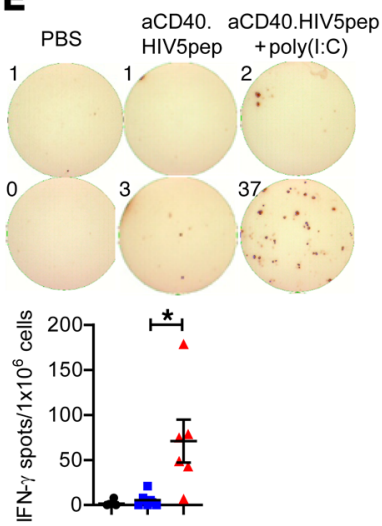

than CpG-B and R848 for enhancing antigen-specific $\mathrm{CD}^{+}$and $\mathrm{CD} 8^{+}$ $\mathrm{T}$ cell responses to vaccination in hu-mice, which correlated with their ability to activate human $\mathrm{mDCs}$ and induce IL-12 (40). In the present study, we tested the therapeutic efficacy of the $\alpha$ CD40.HIV5pep vaccine coadministered with the poly(I:C) adjuvant in vivo in the hu-mice model of persistent HIV-1 infection.

\section{Results}

$\alpha C D 40 . H I V 5 p e p$ plus poly(I:C) vaccination elicits HIV-1-specific human $T$ cell responses. We and others have shown that functional human immune systems, including human DCs and T cells, are developed in immunodeficient mice transplanted with human fetal liver-derived $\mathrm{CD} 4^{+}$cells and thymus, and these hu-mice can initiate $\mathrm{T}$ cell immunity in response to infection or vaccination (41-46). Hu-mice support HIV-1 infection and are proven relevant and robust models to study HIV-1 persistence, pathogenesis, and therapy $(47,48)$. In the present study, we first tested the immunogenicity of the $\alpha$ CD40.HIV5pep vaccine in vivo in hu-mice in the presence of the toll-like receptor 3 (TLR3) agonist poly(I:C) as adjuvant. Poly(I:C) treatment in vivo enhanced the expression of CD40 on human DCs (Figure 1, A and B), which potentially provided more binding targets for the $\alpha \mathrm{CD} 40$.HIV5pep protein. In addition, poly(I:C) treatment also elevated the expression levels of HLA-DR and the costimulatory molecule CD86 on human DCs, which are important for antigen presentation and $\mathrm{T}$ cell activation. We also found that CD141 $1^{+}$DCs, which are important for crosspresentation of exogenous protein antigen to CD ${ }^{+} \mathrm{T}$ cells (49-52), were developed in hu-mice and were activated in vivo by poly $(\mathrm{I}: \mathrm{C})$ treatment (Supplemental Figure 1, A and B; supplemental material available online with this article; https://doi.org/10.1172/ JCI99005DS1). Administration of poly(I:C) in vivo also induced production of human IL-12 and IFN- $\alpha$ (Supplemental Figure 1C), which is important for induction of $\mathrm{T}$ cell immune response.

Thus, we vaccinated hu-mice with the $\alpha$ CD 40.HIV5pep protein with poly(I:C) as adjuvant. Ten days after the boost vaccination, we terminated the mice and detected antigen-specific human $\mathrm{T}$ cell response by stimulating the splenocytes ex vivo with the corresponding pools of 5 HIV-1 long peptides. Without poly(I:C) as adjuvant, $\alpha C D 40$. HIV5pep protein vaccination alone did not induce a significant level of antigen-specific $\mathrm{T}$ cell response (Figure 1, C-E). We found that both $\mathrm{CD}^{+}$and $\mathrm{CD} 4^{+} \mathrm{T}$ cells from 
A

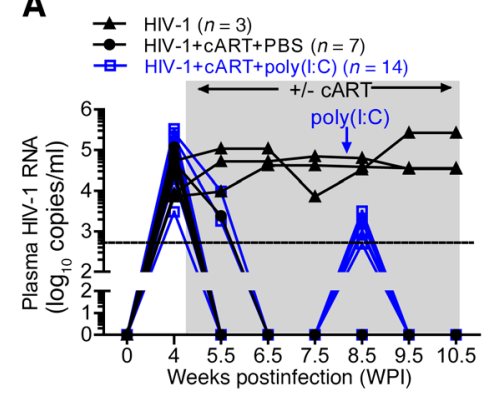

B



Figure 2. Poly(l:C) treatment activates the HIV-1 reservoir in vivo. (A) Hu-mice infected with HIV-1 were treated with CART from 4.5-10.5 weeks postinfection (wpi). At 8 wpi, mice were treated with poly(I:C) or PBS as control. Plasma HIV-1 genome was detected at the indicated time points. Combined data from 2 independent experiments with mean values \pm SEM are shown. (B) Hu-mice were treated as in A. Mice were terminated at 8.5 wpi. Relative cell-associated HIV-1 RNA and DNA in human cells from spleens of HIV-1+CART+PBS-treated mice (PBS), or HIV-1+CART+poly(I:C)-treated mice (poly(I:C)) were quantified by PCR. HIV-1+cART+PBS, $n=4$; HIV- $1+$ CART+poly(I:C), $n=5$. ${ }^{*} P<0.05$ by unpaired, 2-tailed Student's $t$ test.

hu-mice vaccinated with poly(I:C) adjuvant produced IL-2 and TNF- $\alpha$ (Figure 1, C and D) after HIV-1 peptide, but not irrelevant HBV antigen (Supplemental Figure 2) after stimulation ex vivo, indicating vaccination-induced, antigen-specific $\mathrm{T}$ cell responses. $\mathrm{T}$ cells from mice vaccinated with poly(I:C) adjuvant also produced IFN- $\gamma$ after peptide stimulation ex vivo (Figure 1E). Thus, $\alpha C D 40 . H I V 5 p e p$ plus poly(I:C) vaccination elicits HIV-1-specific human $\mathrm{T}$ cell responses in vivo.

Poly(I:C) reactivates $H I V-1$ reservoirs ex vivo in $C D 4^{+} T$ cells from $H I V-1$-infected individuals treated with $c A R T$ and in vivo in infected hu-mice. HIV-1 persists during effective cART in part because its genome becomes stably integrated into latently infected cells. These latently infected cells do not express viral proteins and hence remain invisible to the immune system. We have reported before that, as in humans, cART efficiently suppresses HIV-1 replication in hu-mice, but cells harboring HIV-1 DNA persist (45). It is believed that to eliminate the viral reservoir, latent virus in infected cells needs to be reactivated to express HIV-1 proteins $(53,54)$. TLR agonists are potential reagents to reactivate HIV-1 expression (55-58). Thus, we tested whether the TLR3 agonist poly(I:C), in addition to its immune adjuvant activity, can activate the HIV-1 reservoir in vivo in infected hu-mice under cART. As shown in Figure 2A, cART treatment suppressed HIV-1 viremia in all infected hu-mice within 2 weeks. We treated infected hu-mice with poly(I:C) 3.5 weeks after initiating cART. Interestingly, poly(I:C) treatment in the presence of cART led to low "blips" of HIV-1 viremia within 3 days, which returned to undetectable levels after 1 week (Figure 2A). We detected increased levels of cell-associated HIV-1 RNA but not cell-associated HIV-1 DNA (Figure 2B) at the time point of virus rebound (8.5 weeks after infection), which suggested that the low blips of viremia in the plasma under cART were due to more active HIV-1 transcription after poly(I:C) treatment.

We further tested whether TLR3 agonist poly(I:C) can reactivate HIV-1 reservoirs in CD4 ${ }^{+} \mathrm{T}$ cells from cART-treated, HIV-1infected individuals by virus outgrowth assay. We cultured resting memory $\mathrm{CD}^{+}{ }^{+} \mathrm{T}$ cells of cART-treated individuals with undetectable plasma HIV-1 RNA (below 20 copies/ml) with autologous CD3-depleted blood mononuclear cells either irradiated or not irradiated in the presence of poly $(\mathrm{I}: \mathrm{C})$ as previously described (59). As controls, cells were stimulated with anti-CD3/anti-CD28 monoclonal antibody (mAb) (positive control) or left unexposed (negative control). Supernatants were collected at day 14 for HIV-1 RNA detection. The data indicated that poly(I:C) at either $5 \mu \mathrm{g} /$ $\mathrm{ml}$ or $10 \mu \mathrm{g} / \mathrm{ml}$ induced significant production of HIV-1 RNA from $5 \times 10^{5}$ resting memory $\mathrm{CD}^{+} \mathrm{T}$ cells in the culture supernatants compared with untreated cultures (Figure 3, A and B). Compared with the anti-CD3/anti-CD28 mAb, the efficiency of reactivation of HIV-1 with poly(I:C) was around $20 \%$ at $5 \mu \mathrm{g} / \mathrm{ml}$, and around $35 \%$ at $10 \mu \mathrm{g} / \mathrm{ml}$ by virus outgrowth assay (Figure 3, C and D). No difference was observed on the effect of HIV-1 reservoir reactivation from resting memory CD4 by poly(I:C) when cocultured with autologous CD3-depleted blood mononuclear cells either irradiated or not irradiated, suggesting that HIV-1 RNA was reactivated from resting memory $\mathrm{CD} 4^{+} \mathrm{T}$ cells (Figure 3, A-D). In addition, we also stimulated total leukocytes from lymphoid organs of HIV-1infected hu-mice under cART ex vivo with poly(I:C) or with other TLR agonists. We found that poly(I:C) worked as efficiently as the TLR7 agonists R837 and R848 to reactivate HIV-1 RNA (Figure $3 \mathrm{E}$ ), whereas CpG-B did not reactivate HIV-1 RNA compared with PBS-treated control (Figure 3E). As positive control, the inhibitor of histone deacetylases (SAHA) reactivated HIV-1 RNA more efficiently (10.4-fold more than control) than poly(I:C) (2.3-fold more than control) (Figure 3E). No change of cell-associated HIV-1 DNA was detected after stimulation with TLR agonists, suggesting that the increase in cell-associated RNA was due to more active HIV-1 transcription, but not new infection during the culture (Figure $3 \mathrm{~F}$ ).

In summary, we found that in addition to its adjuvant activity in enhancing anti-HIV-1 T cell response to vaccination, poly(I:C) can also function as a reservoir-activating agent.

$\alpha C D 40 . H I V 5 p e p$ with poly(I:C) vaccination rescues anti-HIV-1 T cell responses and reduces the size of the $H I V-1$ reservoir. We have previously reported that persistent HIV-1 infection in hu-mice led to T cell tolerance, and cART treatment rescued the number but not the function of T cells $(45,46)$. We next determined whether $\alpha \mathrm{CD} 40$. HIV5pep plus poly(I:C) vaccination could rescue anti-HIV-1 T cell activity in infected hu-mice under cART. Hu-mice infected with HIV-1 received cART at 5 weeks postinfection (wpi) through 12 wpi. Hu-mice were vaccinated 2 times with $\alpha$ CD40.HIV5pep plus poly(I:C), at 8 wpi and 11 wpi (Figure 4A). Hu-mice treated with PBS or poly(I:C) alone were used as control. We monitored HIV-1 viremia during the treatment and found that $\alpha$ CD40.HIV5pep with poly(I:C) activated the HIV-1 reservoir in the same manner as treatment with poly(I:C) treatment alone (Figure 4A). At 12 wpi we terminated the hu-mice and determined anti-HIV-1 T cell response levels as well as HIV-1 reservoir size in lymphoid organs. Results indicated that $\alpha \mathrm{CD} 40$.HIV5pep with poly(I:C) rescued the ability of $\mathrm{CD}^{+} \mathrm{T}$ cells to produce both IFN- $\gamma$ and TNF- $\alpha$ in response to HIV-1 peptide stimulation ex vivo (Figure $4, \mathrm{~B}$ and $\mathrm{C}$ ). The IFN- $\gamma$ - and TNF- $\alpha$-expressing T cells also coexpressed the CD107a marker, signifying their killing ability (Supplemental Figure 3). 
A



D

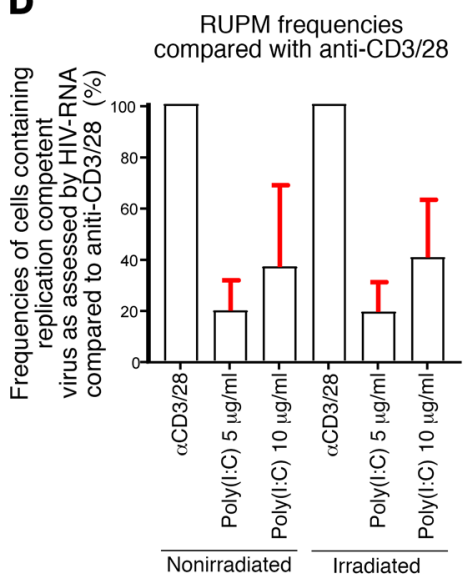

B

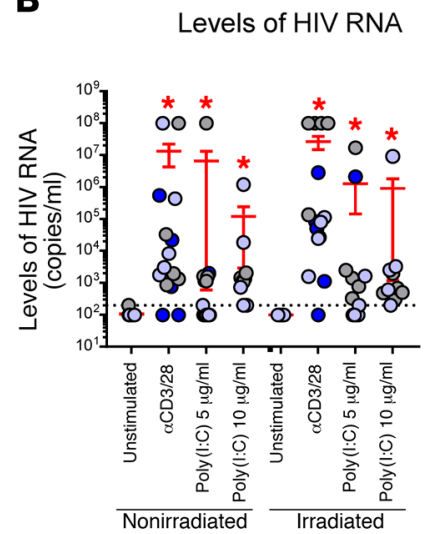

E

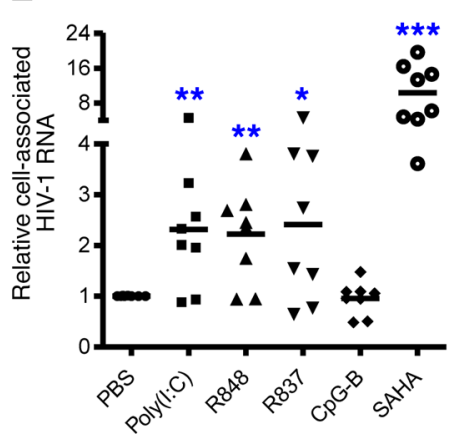

C
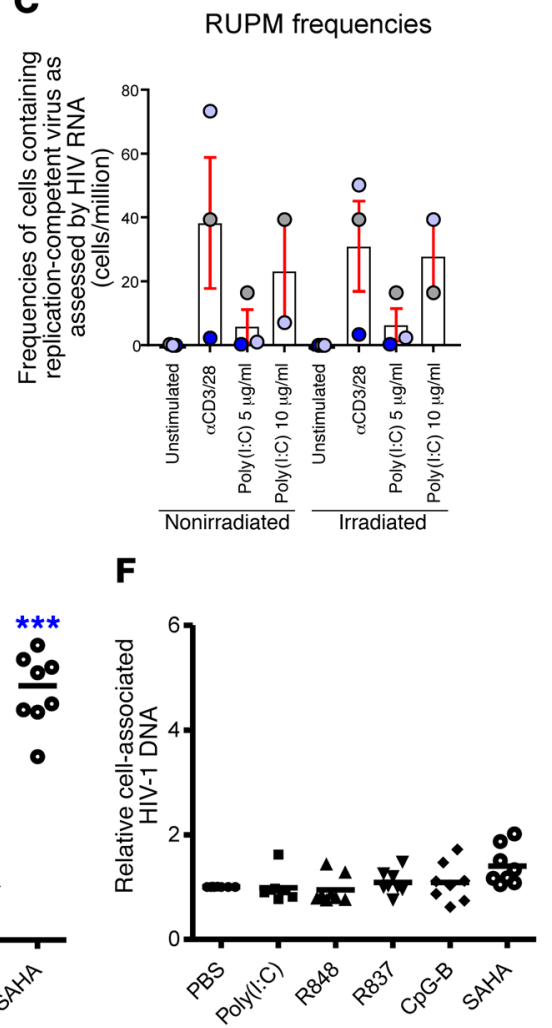

Figure 3. Poly(I:C) treatment reactivates HIV-1 production in cART-treated HIV-1-infected individuals. (A-D) Resting memory CD4+ T cells (HLA-DRCD25 ${ }^{-}$(D69-) of 3 aviremic ART-treated HIV-1-infected individuals were used for virus outgrowth assay. (A) Proportion of HIV-1 RNA-positive wells induced following poly(I:C) treatment. Wells with detectable HIV-1 RNA ( $\geq 200$ HIV-1 RNA copies/ml) were referred to as HIV-1 RNA-positive wells for the condition tested. (B) Levels of HIV-1 RNA copies/ml induced following poly(l:C) treatment. (C) Frequencies of cells containing inducible replication-competent virus as measured by RUPM induced upon poly(I:C) treatment. (D) Fraction of poly(I:C)-induced cells containing replication-competent virus as assessed by HIV-1 RNA as compared with anti-CD3/28. Panels A-B were generated using the 5 replicates of the highest concentration of cells ( $5 \times 10^{5}$ cells) of all conditions by VOA in 3 aviremic cART-treated HIV-1-infected individuals, except the conditions with poly(I:C) at $10 \mu \mathrm{g} / \mathrm{ml}$, which were generated using 2 aviremic ARTtreated HIV-1-infected individuals. Subjects were color-coded and each color corresponds to a subject (B and $\mathbf{C})$. Histograms correspond to mean (C and $\mathbf{D})$ and red bars correspond to SEM (B-D). Red asterisks indicate statistical significance as compared with the unstimulated or unexposed condition ( $\left.{ }^{*} P<0.05\right)$. Anti-CD3/anti-CD28 MAb is represented by 3/28. Statistical significance was obtained using either 2-tailed Chi-square analysis for comparison of positive proportions (A) or 1-way ANOVA (Kruskal-Wallis test) followed by Wilcoxon matched-pairs 2-tailed signed rank test (B-D). (E and F) Combined cells from spleen, bone marrow, and $\mathrm{mLN}$ of cART-treated HIV-1-infected hu-mice were cultured ex vivo with poly(l:C) $(5 \mu \mathrm{g} / \mathrm{ml}), \mathrm{R} 848(5 \mu \mathrm{g} / \mathrm{ml}), \mathrm{R} 837(5 \mu \mathrm{g} / \mathrm{ml}), \mathrm{CpG}-\mathrm{B}$ $(5 \mu \mathrm{g} / \mathrm{ml})$, and SAHA $(1 \mu \mathrm{M})$ in the presence of the antiretroviral drug nevirapine. Each dot represents data from 1 mouse. Relative cell-associated HIV-1 RNA (E) and DNA (F) were detected 48 hours after culture. ${ }^{* *} P<0.01,{ }^{* *} P<0.001$ by unpaired, 2 -tailed Student's $t$ test.

Therapeutic vaccination also rescued the ability of $\mathrm{CD} 4^{+} \mathrm{T}$ cells to produce TNF- $\alpha$ in response to stimulation of specific peptides ex vivo (Figure 4, B and C). Poly(I:C) treatment alone, although it activated the HIV-1 reservoir (Figure 4A), did not enhance anti-HIV-1 T cell activity (Figure 4, B and C).

We next investigated whether therapeutic vaccination reduced the HIV-1 reservoir size in infected hu-mice under cART. We found that cell-associated HIV-1 DNA was significantly decreased by 7.6fold in splenocytes, 10.2-fold in cells from bone marrow, and 13.8fold in cells from mesenteric lymph nodes (mLNs) in vaccinated hu-mice compared with PBS-treated control hu-mice (Figure 4D and Table 1). Cell-associated HIV-1 RNA was also reduced in spleen (8-fold), bone marrow (3.6-fold), and mLNs (7-fold) (Figure 4E and Table 1). Poly(I:C) treatment alone did not change the HIV-1 reservoir size as detected by cell-associated HIV-1 DNA or RNA (Fig- ure 4, D and $\mathrm{E}$ and Table 1). We performed a correlation analysis and found that anti-HIV-1 $\mathrm{CD} 8^{+}$and $\mathrm{CD}^{+}{ }^{+} \mathrm{T}$ cell responses were reversely associated with HIV-1 DNA and RNA levels in different lymphoid organs (Supplemental Figure 4). Thus, aCD40.HIV5pep with poly(I:C) vaccination, in the presence of cART, enhances antiHIV-1 T cell activity and reduces the HIV-1 reservoir.

Hu-mice vaccinated with $\alpha$ CD40.HIV5pep plus poly(I:C) show delayed rebound of virus replication after cART interruption. Finally, we analyzed the effect of $\alpha \mathrm{CD} 40$.HIV5pep plus poly(I:C) vaccination on HIV-1 rebound after cART discontinuation. Hu-mice with persistent HIV-1 infection received cART followed by 2 vaccinations after full suppression of HIV-1 (Figure 5A). We stopped cART at 11 wpi (1 week after the last vaccination) and monitored virus rebound after cART (Figure 5A). One week after cART cessation (12 wpi), HIV-1 rebounded in 14\% (1/7) of control-treated and 29\% 
A

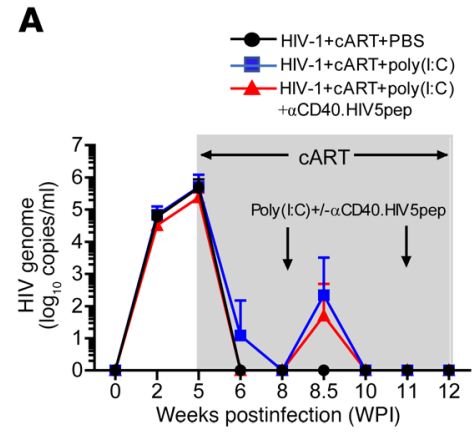

B

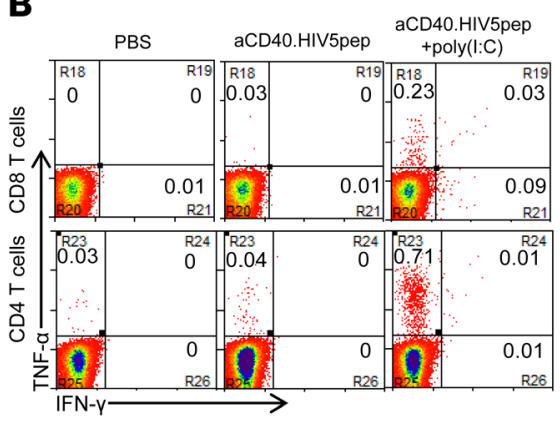

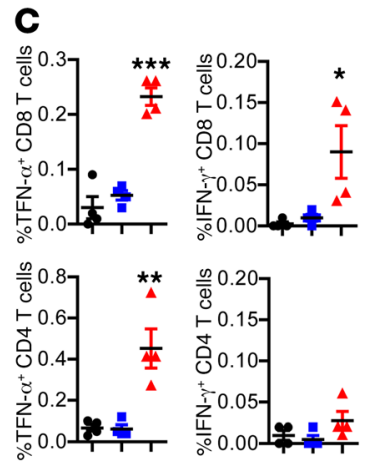

D

$\mathbf{E}$
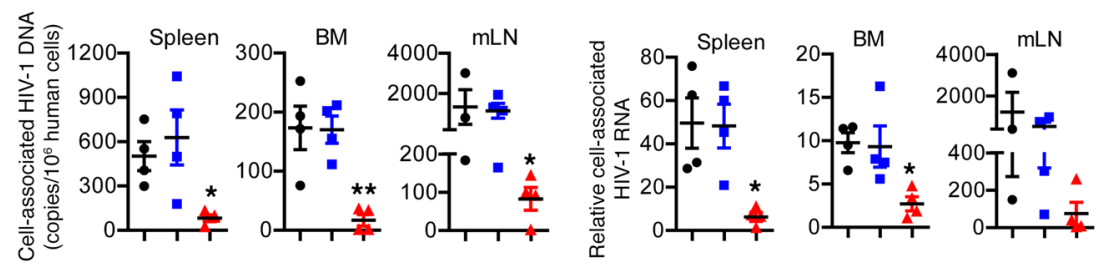

Figure 4. $\alpha$ CD40.HIV5pep plus poly $(\mathrm{I}: \mathrm{C})$ therapeutic vaccination rescues anti-HIV-1 T cell responses and reduces the size of HIV-1 reservoirs. Hu-mice infected with HIV-1 were treated with cART from 5 wpi to 12 wpi. The mice were vaccinated with $\alpha$ CD40.HIV5pep plus poly(I:C) or treated with poly(I:C) or PBS as control at 8 wpi and 11 wpi. Mice were terminated at 12 wpi. (A) Plasma HIV-1 RNA in each group at the indicated time point. (B and C) At termination, splenocytes from mice were stimulated ex vivo with corresponding 5 specific HIV-1 long peptides plus $\alpha$ CD28 mAb for 8 hours (BFA added at 3 hours). Representative plots (B) and summarized data (C) show percentages of IFN- $\gamma^{+}$and TNF- $\alpha^{+}$CD8 $8^{+}$and CD4 $4^{+}$T cells. (D) Cell-associated HIV-1 DNA in human cells from spleen, bone marrow, and mLNs was quantified by PCR. (E) Relative levels of cell-associated HIV-1 RNA in human cells from spleens, bone marrow, and $\mathrm{mLNs}$ were quantified by PCR. (A and C-E) Mean \pm SEM from 4 mice in each group. ${ }^{*} P<0.05,{ }^{* *} P<0.01,{ }^{* * *} P<0.001$ by 1 -way ANOVA and Bonferroni's post hoc test.

(2/7) of poly(I:C)-treated hu-mice, but did not rebound in the vaccinated hu-mice (Figure 5, B-D and Table 2). By the second week (13 wpi), $100 \%$ (7/7) of the control-treated and 100\% (7/7) of the poly(I:C)-treated hu-mice became HIV-1-positive, whereas only $25 \%(2 / 8)$ of vaccinated hu-mice showed detectable viremia in the blood (Figure 5, B-D and Table 2). We detected HIV-1 rebound in all vaccinated hu-mice (8/8) by the third week after cART cessation (14 wpi); however, the viremia in those hu-mice with rebounded HIV-1 was significantly lower in the vaccinated group than in the control groups (Figure 5, B-D and Table 2). By the fourth week (15 wpi), the vaccinated mice still showed lower viral load in the plasma compared with the control mice (Figure 5, B-D and Table 2).

Based on the findings described above, we conclude that $\alpha C D 40$.HIV5pep with poly(I:C) vaccination rescues anti-HIV-1 T cell responses, reduces cART-resistant HIV-1 reservoirs, and leads to better control of virus replication after discontinuation of cART.

\section{Discussion}

In humans, cART suppresses HIV-1 replication but is not curative due to the remarkably long half-life of the HIV-1 reservoir. One approach that has generated considerable enthusiasm is combining strategies to induce the production of virus from latently infected cells together with interventions that can enhance the ability of the host immune system to clear the virus-producing cells $(53,54)$. Using the humanized mouse model of persistent HIV-1 infection, we show here that a therapeutic vaccine targeting HIV-1 antigens to CD40 combined with poly(I:C) activated the cART-resistant HIV-1 reservoir, enhanced anti-HIV-1 T cell response, and thereby reduced the HIV-1 reservoir size.
Targeting of vaccine antigen to DCs is a promising strategy for boosting vaccine immunogenicity and inducing protective or therapeutic efficacy (18). We have shown before that $\alpha \mathrm{CD} 40$. HIV5pep can effectively expand HIV-1 antigen-specific multifunctional helper $\mathrm{CD}^{+}$and cytotoxic $\mathrm{CD}^{+} \mathrm{T}$ cells in the PBMCs of HIV-infected patients, and the expanded polyfunctional cytotoxic $\mathrm{CD}^{+} \mathrm{T}$ cells can control HIV-1 replication in vitro (39). Here we take advantage of hu-mice with functional human immune systems to test both the immunogenicity and efficacy of the aCD40.HIV5pep vaccine in vivo. We also tested the TLR3 agonist to induce CD40 expression on DCs to enhance the efficacy of the $\alpha \mathrm{CD} 40 . \mathrm{HIV} 5$ pep vaccine. We found that $\alpha \mathrm{CD} 40$.HIV5pep with poly(I:C) vaccination elicits both functional HIV-1 antigen-specific human $\mathrm{CD} 8^{+}$and $\mathrm{CD} 4^{+}$responses in vivo in naive hu-mice. Importantly, when administrated in a therapeutic setting in cART-suppressed mice, $\alpha \mathrm{CD} 40$. HIV5pep with poly(I:C) vaccination restored the $\mathrm{CD} 8^{+} \mathrm{T}$ cells to produce IFN- $\gamma$ and TNF- $\alpha$ in response to HIV- 1 antigen stimulation. Our promising results in hu-mice provide the preclinical rationale to test the immunogenicity of the $\alpha \mathrm{CD} 40$.HIV5pep vaccine in healthy volunteers and the therapeutic benefit of the vaccine to reverse anti-HIV-1 T cell function in HIV-1-infected patients.

It has been proposed that HIV-1 therapeutic vaccination should be combined with reservoir-activating agents. TLR agonists are potent vaccine adjuvants (60) and may also activate the HIV-1 reservoir or reverse HIV-1 latency (55-57). Whitney et al. recently reported that a TLR7 agonist given to SIV-infected ART-suppressed Rhesus macaques caused transient increases in the level of plasma virus (58). We found here that the TLR3 agonist poly(I:C) treatment led to transient production of plasma HIV-1 RNA blips 3 days after 
Table 1. $\alpha C D 40$. HIV5pep with poly(I:C) vaccination in the presence of CART reduces the size of the HIV-1 reservoir

\begin{tabular}{|c|c|c|c|c|c|c|c|c|c|c|c|c|c|}
\hline \multirow[t]{2}{*}{ Donor } & \multirow[t]{2}{*}{ Mouse no. } & \multicolumn{2}{|c|}{$\begin{array}{l}\text { Percentage of cell } \\
\text { (preinfection) }\end{array}$} & \multirow[t]{2}{*}{ Treatment } & \multicolumn{5}{|c|}{ HIV-1 RNA in plasma $\left(\log _{10}\right)$} & \multicolumn{4}{|c|}{ Cell-associated DNA/RNA } \\
\hline & & & & & 5 wpi & 8 wpi & 8.5 wpi & 10 wpi & 12 wpi & $\mathrm{DNA}^{\mathrm{C}}$ & $\mathrm{RNA}^{\mathrm{D}}$ & DNA & RNA \\
\hline 1 & 2776 & 80.5 & 76.8 & PBS & 6.0 & ND & ND & ND & ND & 407 & 76 & 253 & 11 \\
\hline 1 & 2777 & 49.3 & 75.2 & PBS & 5.9 & ND & ND & ND & ND & 551 & 29 & 194 & 9.5 \\
\hline 1 & 2764 & 65.1 & 44.9 & PBS & 5.6 & ND & ND & ND & ND & 298 & 32 & 171 & 6.6 \\
\hline 1 & 2747 & 84.3 & 81.9 & Poly(l:C) & 5.4 & ND & 3.3 & ND & ND & 450 & 21 & 202 & 7.9 \\
\hline 1 & 2756 & 82.1 & 73.7 & Poly(l:C) & 6.5 & ND & ND & ND & ND & 180 & 67 & 155 & 16 \\
\hline 1 & 2757 & 60.5 & 72.4 & Poly(I:C) & 5.7 & ND & ND & ND & ND & 1043 & 60 & 111 & 7.5 \\
\hline 1 & 2751 & 39.9 & 70.9 & Vax & 5.3 & ND & ND & ND & ND & 95 & 6.8 & ND & 1.0 \\
\hline 1 & 2752 & 89.9 & 79.5 & Vax & 5.6 & ND & ND & ND & ND & 21 & 11 & 33 & 4.7 \\
\hline 1 & 2754 & 90.2 & 80.2 & Vax & 5.0 & ND & 3.5 & ND & ND & 129 & 6.2 & 35 & 1.8 \\
\hline
\end{tabular}

Humanized mice engrafted with human HSC and thymus were treated as in Figure 3A. APercentage of human CD45+ of total cells in PBMCs. ${ }^{\mathrm{B} P e r c e n t a g e}$ of $\mathrm{CD}^{+}$from human $\mathrm{CD} 45^{+}$cells. ${ }^{\complement}$ Value stands for copies per $10^{6}$ human cells. ${ }^{D}$ Value stands for relative level as defined in Methods. ${ }^{\mathrm{E} D o n o r} \mathrm{HLA}$ class I type: HLA-A2', HLA-B7,27- HLA-B57,58- wpi, weeks postinfection. Vax, vaccination with aCD40.HIV5pep+poly(I:C). ND, not detectable.

treatment in HIV-1-infected mice with cART treatment. This result indicates that poly(I:C) may work as a reservoir-activating agent in vivo. HIV-1 RNA in plasma became undetectable 1 week after treatment, probably due to inhibition by cART of reactivated HIV-1 replication. In addition, we proved that poly(I:C) can reactivate HIV-1 RNA from resting memory $\mathrm{CD}^{+}{ }^{+} \mathrm{T}$ cells from cART-treated patients. Together, our data support the concept that poly(I:C) can potentially function as an HIV-1 reservoir-activating agent. The advantage of using TLR agonists as reservoir-activating agents is that they are also potent vaccine adjuvants, whereas HDAC inhibitors suppress host cellular immune functions $(61,62)$.

A key observation from our study is that $\alpha$ CD40.HIV5pep with poly(I:C) vaccination led to reduction of HIV-1 reservoir size in spleen and bone marrow and delayed virus rebound after cART discontinuation in HIV-1-infected cART-suppressed hu-mice. Of note, poly $(\mathrm{I}: \mathrm{C})$ treatment alone failed to reduce the HIV-1 reservoir or delay virus rebound, although it activated the HIV-1 reservoir in vivo. These data suggest that the anti-HIV-1 T cell response elicited or rescued by the vaccination contributed to the reservoir reduction and virus control after cART interruption. We also observed that even though the virus rebounded in all vaccinated mice at later time points after stopping cART, the plasma HIV-1 RNA levels were lower in vaccinated hu-mice compared with the PBSor poly(I:C)-treated mice, indicating that vaccination enhanced virus control. It is important to point out that some cohorts of hu-mice that we used were reconstituted with HSCs from HLA$\mathrm{B} 57 / \mathrm{B}^{+} 8^{+}$donors, which may enhance vaccine responsiveness. It will be of interest to compare the relative response to HIV-1 vaccination in hu-mice with HLA-B57/B58 ${ }^{+}$and HLA-B57/B58- donors in future studies. It is also important to state that there are several limitations of the humanized mouse model, including short-term cART of HIV-1 infection and infection with a molecular clone of HIV-1. In addition, the human immunity developed in hu-mice is not fully functional as that found in immunocompetent hosts (42,
47). The lymphoid structures, such as B cell follicles, which have been identified as HIV-1 sanctuary sites for HIV-1 reservoir cells to escape from $\mathrm{CD}^{+}$cell-mediated killing in nonhuman primate models (63) and HIV-1-infected people (64), are not fully developed in hu-mice. The restored anti-HIV-1 T cell immune response by vaccination in hu-mice may not be robust enough to eliminate or fully control the HIV-1 reservoir. We believe that the effect of the vaccination would be better in immunocompetent hosts. We believe that our proof-of-concept study in hu-mice has important implication for the development of CD40-targeting HIV-1 vaccine to cure HIV-1 infection in humans.

\section{Methods}

Construction of hu-mice. NRG (NOD-Rag2 $2^{-/} \gamma \mathrm{c}^{-/-}$) mice were obtained from the Jackson Laboratory. All mice were housed and bred in a specific pathogen-free environment. Hu-mice were generated as previously reported (65). Briefly, 6- to 8-week-old NRG mice were sublethally irradiated and anesthetized, and $1-\mathrm{mm}^{3}$ fragments of human fetal thymus were implanted under the kidney capsule. CD $34^{+}$hematopoietic progenitor cells purified from fetal liver of the same donor were injected intravenously within 3 hours. Human immune cell engraftment was detected by flow cytometry 12 weeks after transplantation. We used different human donors for different cohorts of hu-mice in the study.

Production of $\alpha$ CD40.HIV5pep protein and vaccination. Recombinant anti-human CD40 antibody fused to 5 HIV peptide regions ( $\alpha$ CD40.HIV5pep) was produced as previously reported (39), except that we humanized the mouse variable regions to reduce antigenicity in humans and relocated 2 of the HIV5pep regions onto the light chain C-terminus to improve productivity. These sequences, hAntiCD4OVH3-LV-hIgG4H-C-Flex-v1-Pepgag17-f1-gag253-f2-nef116-f3 (GenBank KM660791) and hAnti-CD40VK2-LV-hIgGKC-pol158f3-nef66 (GenBank KM660792), were configured in vectors and expressed and purified as protein secreted from stably transfected 
A

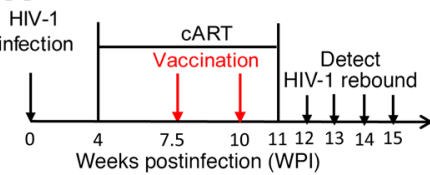

C

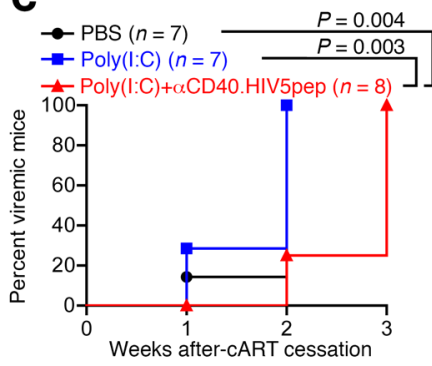

B

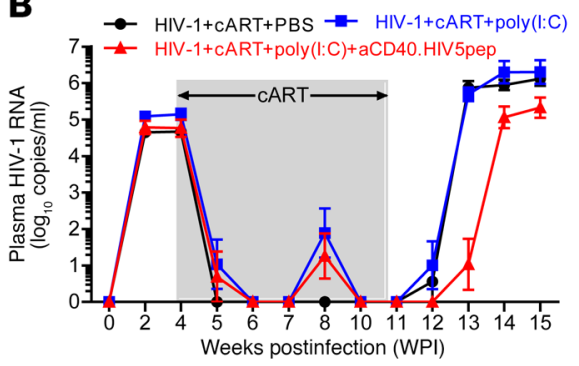

D

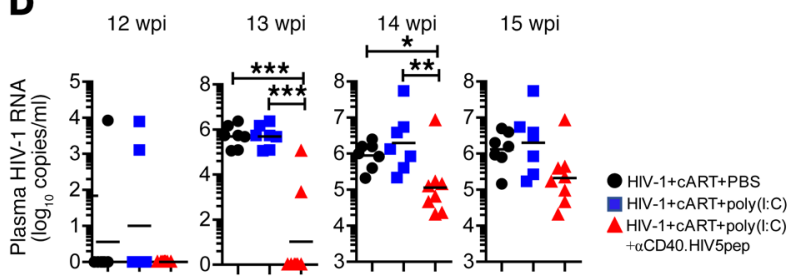

Figure 5. Hu-mice receiving $\alpha \mathrm{CD} 40$.HIV5pep plus poly(l:C) therapeutic vaccination show improved control of HIV-1 replication after cART discontinuation. (A) Schematic diagram of the experimental design. Hu-mice infected with HIV-1 were treated with cART from 4 wpi to 11 wpi. The mice were vaccinated with $\alpha$ CD40.HIV5pep plus poly(I:C) or treated with poly(I:C) or PBS as control at 7.5 wpi and 10 wpi. Virus rebound was detected by PCR weekly after CART cessation at 11 wpi. (B) Plasma HIV-1 RNA in each group at indicated time points. (C) Kinetic analysis of HIV-1 rebound after cART cessation. (D) Plasma HIV-1 RNA at 12, 13, 14, 15 wpi from each mouse in different treatment groups. (B-D) Combined data from 2 independent experiments with mean

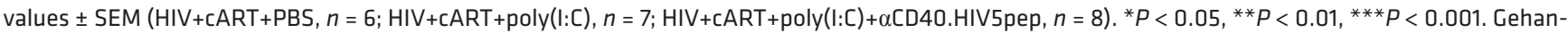
Breslow-Wilcoxon test (C) or 1-way ANOVA and Bonferroni's post hoc test (D) was performed.

CHO-S cells as previously described (39). The vaccines had low lipopolysaccharide levels of $0.04-0.07 \mathrm{ng} / \mathrm{mg}$ protein. For vaccination, hu-mice were intramuscularly (half-dose) and intraperitoneally (halfdose) injected with $10 \mu \mathrm{g} \alpha \mathrm{CD} 40$-HIV5pep alone or coinjected with $50 \mu \mathrm{g}$ poly(I:C). Control mice received PBS or poly(I:C) treatment.

HIV-1 infection of hu-mice. The R5 tropic strain of HIV-1 (JR-CSF) was generated by transfection of $293 \mathrm{~T}$ cells with plasmid containing full-length HIV-1 (JR-CSF) genome. Hu-mice with stable human leukocyte reconstitution were anesthetized and infected with HIV-1 (JR-CSF) (10 ng p24 or 3,000 infectious units per mouse) through retro-orbital injection. Both male and female mice were used for all the experiments.

Combination antiretroviral therapy (CART). Food formulated with antiretroviral individual drugs was prepared as reported with elevated dose modifications (66). In brief, tablets of emtricitabine and tenofovir disoproxil fumarate (Truvada; Gilead Sciences) and raltegravir (Isentress; Merck) were crushed into fine powder and manufactured with TestDiet 5B1Q feed (Modified LabDiet 5058 with $0.12 \%$ amoxicillin) into half-inch irradiated pellets. Final concentrations of drugs in the food were $4,800 \mathrm{mg} / \mathrm{kg}$ raltegravir, $1,560 \mathrm{mg} / \mathrm{kg}$ tenofovir disoproxil, and $1,040 \mathrm{mg} / \mathrm{kg}$ emtricitabine. The estimated daily drug doses were $768 \mathrm{mg} / \mathrm{kg}$ raltegravir, 250 $\mathrm{mg} / \mathrm{kg}$ tenofovir disoproxil, and $166 \mathrm{mg} / \mathrm{kg}$ emtricitabine.

HIV-1 genomic RNA detection in plasma. HIV-1 RNA was purified from the plasma with the QIAampkit Viral RNA Mini Kit (Qiagen). The RNA was then reverse transcribed and quantitatively detected by real-time PCR using the TaqMan Fast Virus 1-Step PCR kit (Thermo Fisher Scientific). The primers used for detecting the HIV Gag gene were (5'- GGTGCGAGAGCGTCAGTATTAAG-3' and 5'AGCTCCCTGCTTGCCCATA-3'). The probe (FAM-AAAATTCGGTTAAGGCCAGGGGGAAAGAA-QSY7) used for detection was ordered from Applied Biosystems and the reactions were set up following manufacturer's guidelines and were run on the QuantStudio 6 Flex PCR system (Applied Biosystems). The detection limit of the real-time PCR reaction is 4 copies per reaction. Accordingly, due to the relatively small volume of each bleeding in mice (around 50-100 $\mu$ l total blood), the limit of detection of the assay is 400 copies/ml plasma. We set the copy number that is below the detectable limit as 1 .

Cell-associated HIV-1 DNA detection. To measure total cellassociated HIV-1 DNA, nucleic acid was extracted from spleen and bone marrow cells using the DNeasy mini kit (Qiagen). HIV-1 DNA was quantified by real-time PCR. DNA from serial dilutions of ACH2 cells, which contain 1 copy of the HIV genome in each cell, was used to generate a standard curve.

Cell-associated HIV-1 RNA detection. To measure total cellassociated HIV-1 RNA, nucleic acid was extracted from spleen or bone marrow cells using the RNeasy plus mini kit (Qiagen). HIV-1 RNA was detected as described above. The HIV-1 RNA expression levels were normalized to human CD4 mRNA (5'-GGGCTTCCTCCTCCAAGTCTT-3' and CCGCTTCGAGACCTTTGC) controls and the result was calculated as fold change in gene expression.

Antigen-specific $T$ cell response detection. For antigen-specific stimulation, splenocytes from vaccinated hu-mice were collected 10 days after the second vaccination and stimulated ex vivo with 5 specific HIV long peptides (39) plus $\alpha$ CD28 for 12 hours. Brefeldin A was added during the last 4 hours of stimulation and IL-2/TNF- $\alpha$ expression by $\mathrm{CD}^{+}$and $\mathrm{CD} 8^{+} \mathrm{T}$ cells was detected by intracellular staining. ELISpot plates (96-well, Millipore, catalog S2EM004M99) were coated with $5 \mu \mathrm{g} / \mathrm{ml}$ mouse anti-human IFN- $\gamma$ antibody (BD Pharmingen) overnight at $4^{\circ} \mathrm{C}$. After blocking with complete RPMI for 2 hours at $37^{\circ} \mathrm{C}$, $5 \times 10^{5}$ splenocytes were stimulated with the corresponding 5 specific 
Table 2. $\alpha$ CD40.HIV5pep with poly(I:C) vaccination in the presence of cART delays HIV-1 rebound after cART cessation

\begin{tabular}{|c|c|c|c|}
\hline \multirow[t]{2}{*}{ Donor } & \multirow[t]{2}{*}{ Mouse no. } & \multicolumn{2}{|c|}{$\begin{array}{l}\text { Percentage of cell } \\
\text { (preinfection) }\end{array}$} \\
\hline & & $\operatorname{CD}_{4}{ }^{\mathrm{A}}$ & $\mathrm{CD} 3$ \\
\hline $2^{c}$ & 1527 & 58 & 72. \\
\hline 2 & 1534 & 41 & 74. \\
\hline 2 & 1537 & 76.8 & 30 \\
\hline 2 & 1529 & 23.9 & 29. \\
\hline 2 & 1531 & 48.1 & 57 \\
\hline 2 & 1533 & 33.5 & 56 \\
\hline 2 & 1515 & 54.3 & 17. \\
\hline 2 & 1518 & 75.2 & \\
\hline 2 & 1525 & 41.1 & \\
\hline 2 & 1526 & 63.4 & 82 \\
\hline $3^{0}$ & 1703 & 35.6 & \\
\hline 3 & 1704 & 28.9 & \\
\hline 3 & 1705 & 60.4 & \\
\hline 3 & 1706 & 55.6 & \\
\hline 3 & 1707 & 28.5 & 61. \\
\hline 3 & 1708 & 27.1 & 61 \\
\hline 3 & 1720 & 60.6 & \\
\hline 3 & 1721 & 34.0 & \\
\hline 3 & 1711 & 56.6 & \\
\hline 3 & 1714 & 25.5 & \\
\hline 3 & 1715 & 27.2 & \\
\hline 3 & 1717 & 50.9 & \\
\hline
\end{tabular}

Treatment

\begin{tabular}{|c|c|c|}
\hline & 4 wpi & 7 wpi \\
\hline PBS & 4.8 & ND \\
\hline PBS & 5.1 & ND \\
\hline PBS & 4.5 & ND \\
\hline Poly (l:C) & 5.3 & ND \\
\hline Poly(l:C) & 4.4 & ND \\
\hline Poly(l:C) & 4.8 & ND \\
\hline Vax & 4.3 & ND \\
\hline Vax & 5.0 & ND \\
\hline Vax & 3.5 & ND \\
\hline Vax & 5.3 & ND \\
\hline PBS & 5.3 & ND \\
\hline
\end{tabular}

PBS $\quad 4.9$

PBS $\quad 5.4$

PBS $\quad 5.0$

Poly(l:C) $\quad 5.4$

Poly $(\mathrm{l:C} \quad 5.3$

Poly(I:C) 5.2

Poly(l:C) $\quad 5.5$

Vax 5.3

Vax $\quad 4.3$

Vax 5.3

Vax

HIV-1 RNA in plasma $\left(\log _{10}\right)$

\begin{tabular}{cccccc}
8 wpi & 11 wpi & 12 wpi & 13 wpi & 14 wpi & 15 wpi \\
ND & ND & ND & 5.1 & 5.3 & 5.2 \\
ND & ND & 3.9 & 6.6 & 6.0 & 6.0 \\
ND & ND & ND & 5.9 & 5.9 & 5.9 \\
3.7 & ND & 3.9 & 5.1 & 5.3 & 5.2 \\
3.3 & ND & ND & 5.7 & 5.6 & 5.4 \\
ND & ND & ND & 6.4 & 6.1 & 6.5 \\
ND & ND & ND & ND & 5.1 & 5.3 \\
ND & ND & ND & 5.1 & 4.8 & 5.6 \\
\hline 3.5 & ND & ND & ND & 5.2 & 5.0 \\
\hline 3.3 & ND & ND & ND & 4.4 & 5.6 \\
ND & ND & ND & 6.2 & 6.2 & 6.3 \\
ND & ND & ND & 5.7 & 5.6 & 6.6 \\
ND & ND & ND & 5.7 & 6.2 & 6.2 \\
ND & ND & ND & 5.1 & 6.4 & 6.7 \\
\hline 3.0 & ND & ND & 6.2 & 6.7 & 6.5 \\
ND & ND & ND & 5.7 & 6.6 & 6.9 \\
ND & ND & 3.1 & 5.7 & 7.7 & 7.7 \\
\hline 3.3 & ND & ND & 5.1 & 5.9 & 5.8 \\
\hline ND & ND & ND & ND & 5.2 & 5.3 \\
\hline 3.1 & ND & ND & ND & 4.3 & 4.0 \\
ND & ND & ND & 3.2 & 6.9 & 7.0 \\
ND & ND & ND & ND & 4.7 & 4.9
\end{tabular}

Humanized mice engrafted with human HSC and thymus were treated as in Figure 4A. ${ }^{A}$ Percentage of human CD45 ${ }^{+}$of total cells in PBMCs. ${ }^{\mathrm{B} P e r c e n t a g e}$ of $\mathrm{CD}^{+}$from human CD45+ cells. 'Donor HLA class I type: HLA-A2+, HLA-B7,27-, HLA-B57,58+. ${ }^{\circ}$ Donor HLA class I type: HLA-A2+, HLA-B7,27-, HLA-B57,58+'. wpi, weeks post HIV-1 infection. ND, not detectable. Vax, vaccination with $\alpha$ CD40.HIV5pep plus poly(I:C).

HIV-1 long peptides at $2 \mu \mathrm{g} / \mathrm{ml}$, while addition of medium served as negative control. Plates were incubated at $37^{\circ} \mathrm{C}$ for 24 hours before washing with cold $\mathrm{H}_{2} \mathrm{O}$ twice and 5 times with $\mathrm{PBS}$ containing $0.05 \%$ (vol/vol) Tween 20. Biotinylated anti-human IFN- $\gamma$ antibody (BD Pharmingen) was added at $1 \mu \mathrm{g} / \mathrm{ml}$ for 1 hour at $37^{\circ} \mathrm{C}$ and, after washing, a 1:1,000 dilution of Avidin-HRP (BD Pharmingen) was added for 1 hour at $37^{\circ} \mathrm{C}$. After final washing, stable final substrate solution AEC (3-amino-9-ethylcarbazole) (BD Pharmingen) was added to the plate, incubated for 15 minutes at room temperature, and then the reaction was stopped by thorough rinsing with water. After drying, the number of spots in each well was counted with an automated ELISpot plate reader (CTL Immunospot).

Flow cytometry. For surface staining, single-cell suspensions prepared from spleens of hu-mice were stained with surface markers and analyzed on a CyAn ADP (Dako). For intracellular cytokine staining, cells were first stained with surface markers and then permeabilized with cytofix/cytoperm buffer (BD Bioscience), followed by intracellular staining. FITC-conjugated anti-human CD40, PE-conjugated antihuman CD107a, CD141, PE/Cy5-conjugated anti-human CD4, CD86, CD1c, PE/Cy7-conjugated anti-human CD3, HLA-DR, PB-conjugated anti-human CD4, CD14, IL-2, APC-conjugated anti-human CD11c, TNF- $\alpha$ and APC/Cy7-conjugated anti-human CD45, and PE-conjugated anti-human HLA-A2 were purchased from Biolegend. Pacific orangeconjugated anti-mouse CD45, PE/Texas red-conjugated anti-human CD3, CD8, and LIVE/DEAD Fixable Aqua (LD7) Dead Cell Stain Kit were purchased from Invitrogen. FITC-conjugated anti-HIV-1 p24 was purchased from Beckman Coulter. FITC-conjugated anti-HLA-7,27 and biotinylated anti-HLA-57,58 were purchased from One Lamda, Thermo Fisher Scientific. Data were analyzed using Summit 4.3 software (Dako).

Sorting of resting memory $C D 4^{+} T$ cells. Cryopreserved blood mononuclear cells were thawed, and $\mathrm{CD}^{+} \mathrm{T}$ cells were enriched using the EasySep human $\mathrm{CD}^{+} \mathrm{T}$ cell enrichment kit (StemCell Technologies). $\mathrm{CD} 4^{+} \mathrm{T}$ cells were then stained with an Aqua LIVE/ DEAD stain kit $\left(4^{\circ} \mathrm{C}, 15\right.$ minutes) and then with anti-CD4-FITC, anti-CD45RA-ECD, anti-HLA-DR-PB, anti-CD25-PE-Cy7, and antiCD69-PerCp-Cy5.5 ( $4^{\circ} \mathrm{C}, 25$ minutes), and viable resting memory


sorted using a FACSAria fluorescence-activated cell sorter (Becton Dickinson). In all sorting experiments, the grade of purity of the sorted cell populations was greater than $97 \%$.

Viral outgrowth assay (VOA). Different cell concentrations (5-fold limiting dilutions: $5 \times 10^{5}, 10^{5}, 2 \times 10^{4}$, and $4 \times 10^{3}$ cells) of sorted viable resting memory $\mathrm{CD} 4^{+} \mathrm{T}$ cells $\left(\mathrm{HLA}-\mathrm{DR}^{-} \mathrm{CD} 25^{-} \mathrm{CD} 69^{-}\right)$of 3 aviremic cART-treated HIV-1-infected individuals were cultured with autologous CD3-depleted blood mononuclear cells $\left(10^{6}\right.$ cells $\left./ \mathrm{ml}\right)$ irradiated or not irradiated in the presence of poly(I:C) (5 or $10 \mu \mathrm{g} / \mathrm{ml})$ as previously described (59). As controls, cells were stimulated with anti-CD3/ anti-CD28 mAb-coated plates (10 $\mu \mathrm{g} / \mathrm{ml} ; 72$ hours) (positive control) or left unexposed (negative control) as previously described (59). All cell conditions were cultured in complete RPMI supplemented with IL-2 (50 U/ml) for 14 days. Medium was replaced at day 5 and resupplemented with cytokines and poly(I:C). Supernatants were collected 
at day 14 and the presence of HIV-1 RNA was assessed by COBAS AmpliPrep/TaqMan HIV-1 Test (Roche) following 1:10 medium dilution in basement matrix buffer (RUWAG Handels AG) as previously described (59). RNA-units per million (RUPM) frequencies were calculated by conventional limiting dilution methods using Extreme Limiting Dilution analysis (http://bioinf.wehi.edu.au /software/elda/) as previously described (59).

Statistics. Statistical analyses were performed using GraphPad Prism 5.0 software (GraphPad Software). Experiments were analyzed by 2-tailed Student's $t$ test, or by 1-way ANOVA and Bonferroni's post hoc test or Gehan-Breslow-Wilcoxon test, according to the assumptions of the test, as indicated in the figure legends for each experiment. ${ }^{*} P<0.05,{ }^{* *} P<0.01,{ }^{* * *} P<0.001$. All the data with error bars are mean \pm SEM. A $P$ value less than 0.05 was considered significant.

Study approval. Human fetal liver and thymus tissues (gestational age of 16 to 20 weeks) were obtained from elective or medically indicated terminations of pregnancies, through a nonprofit intermediary working with outpatient clinics (Advanced Bioscience Resources). Informed consent of the maternal donors was obtained in all cases, under regulations governing the clinic. The project was reviewed by the university's Office of Human Research Ethics, which determined that the study with hu-mice does not constitute human subject research as defined under federal regulations [45 CFR 46.102 (d or f) and 21 CFR 56.102(c)(e)(l)]. All animal studies were approved by the University of North Carolina Institutional Animal Care and Use Committee (IACUC ID 14-100). The human study was approved and performed by the institutional review board of Lausanne University Hospital, University of Lausanne (i.e., Centre Hospitalier Universitaire Vaudois), and all subjects gave written informed consent.

\section{Author contributions}

LS, GZ, LC, and YL conceived the study. LC, GZ, GP, MP, and LS designed the experiments. LC, QW, GL, RB, JM, HY, FY, and ZZ performed experiments. LC and LS analyzed and interpreted the data. SZ and GZ provided the aCD45.HIV5pep recombinant protein. LC, GZ, YL, and LS prepared the manuscript.

\section{Acknowledgments}

The study was funded in part by grants from National Institutes of Health (AI109784, DK095962, and AI134631 to LS) and within the Vaccine Research Institute (ANRS/INSERM) HIV vaccine program, and was supported by the Investissements d'Avenir program managed by the ANR under reference ANR-10-LABX-77-01. These funding sources had no direct role in any aspect of the research or article. Matthew Baker (Antitope, Ltd) provided humanization services for the $\alpha \mathrm{CD} 4012 \mathrm{E} 12 \mathrm{mAb}$. We thank Mireille Centlivre for coordinating the study and editing the manuscript. We thank Liqun Chi and Yaxu Wu for technical support, and Lineberger Comprehensive Cancer Center cores, the University of North Carolina flow cytometer core, Division of Laboratory Animal Medicine, and the University of North Carolina Centers for AIDS Research for support.

Address correspondence to: Lishan Su, Room 5208, Masico Hall, 125 Mason Farm Road, Chapel Hill, North Carolina 27514, USA. Phone: 919.966.6654, Email: 1su@med.unc.edu. Or to: Yves Levy, Vaccine Research Institute-VRI, Hôpital Henri Mondor, 51, Avenue du Maréchal de Lattre de Tassigny 94010, Créteil, France. Phone:33.01.49.81.44.42; Email: yves.levy@aphp.fr.
1. Archin NM, Sung JM, Garrido C, Soriano-Sarabia N, Margolis DM. Eradicating HIV-1 infection: seeking to clear a persistent pathogen. Nat Rev Microbiol. 2014;12(11):750-764.

2. Katlama C, et al. Barriers to a cure for HIV: new ways to target and eradicate HIV-1 reservoirs. Lancet. 2013;381(9883):2109-2117.

3. Pantaleo G, Levy Y. Therapeutic vaccines and immunological intervention in HIV infection: a paradigm change. Curr Opin HIV AIDS. 2016;11(6):576-584.

4. Deeks SG. HIV infection, inflammation, immunosenescence, and aging. Annu Rev Med. 2011;62:141-155.

5. Cao Y, Qin L, Zhang L, Safrit J, Ho DD. Virologic and immunologic characterization of long-term survivors of human immunodeficiency virus type 1 infection. N Engl J Med. 1995;332(4):201-208.

6. Pantaleo G, et al. Studies in subjects with long-term nonprogressive human immunodeficiency virus infection. NEngl J Med.1995;332(4):209-216.

7. Lambotte O, et al. HIV controllers: a homogeneous group of HIV-1-infected patients with spontaneous control of viral replication. Clin Infect Dis. 2005;41(7):1053-1056.

8. Rosenberg ES, et al. Vigorous HIV-1-specific CD4+ T cell responses associated with control of viremia. Science. 1997;278(5342):1447-1450.

9. Migueles SA, et al. HIV-specific CD8+ T cell proliferation is coupled to perforin expression and is maintained in nonprogressors. Nat Immu- nol. 2002;3(11):1061-1068.

10. Deeks SG, Walker BD. Human immunodeficiency virus controllers: mechanisms of durable virus control in the absence of antiretroviral therapy. Immunity. 2007;27(3):406-416.

11. Letvin NL, Walker BD. Immunopathogenesis and immunotherapy in AIDS virus infections. Nat Med.2003;9(7):861-866.

12. Barouch DH, Deeks SG. Immunologic strategies for HIV-1 remission and eradication. Science. 2014;345(6193):169-174.

13. Mylvaganam GH, Silvestri G, Amara RR. HIV therapeutic vaccines: moving towards a functional cure. Curr Opin Immunol. 2015;35:1-8.

14. Mellman I, Steinman RM. Dendritic cells: specialized and regulated antigen processing machines. Cell. 2001;106(3):255-258.

15. Steinman RM, Banchereau J. Taking dendritic cells into medicine. Nature. 2007;449(7161):419-426.

16. Palucka K, Banchereau J. Dendritic-cellbased therapeutic cancer vaccines. Immunity. 2013;39(1):38-48.

17. Bonifaz L, Bonnyay D, Mahnke K, Rivera M, Nussenzweig MC, Steinman RM. Efficient targeting of protein antigen to the dendritic cell receptor DEC-205 in the steady state leads to antigen presentation on major histocompatibility complex class I products and peripheral CD8+ T cell tolerance. J Exp Med. 2002;196(12):1627-1638.

18. Cohn L, Delamarre L. Dendritic cell-targeted vaccines. Front Immunol. 2014;5:255.
19. Kastenmüller W, Kastenmüller K, Kurts C, Seder RA. Dendritic cell-targeted vaccines--hope or hype? Nat Rev Immunol. 2014;14(10):705-711.

20. Flynn BJ, et al. Immunization with HIV Gag targeted to dendritic cells followed by recombinant New York vaccinia virus induces robust T-cell immunity in nonhuman primates. Proc Natl Acad Sci USA. 2011;108(17):7131-7136.

21. Flamar AL, et al. Delivering HIV Gagp24 to DCIR Induces Strong Antibody Responses In Vivo. PLOS ONE. 2015;10(9):e0135513.

22. Zurawski G, et al. Targeting HIV-1 Env gp140 to LOX-1 Elicits Immune Responses in Rhesus Macaques. PLoS ONE. 2016;11(4):e0153484.

23. Zurawski G, et al. Superiority in Rhesus macaques of targeting HIV-1 Env gp140 to CD40 versus LOX-1 in combination with replication-competent NYVAC-KC for induction of Env-specific antibody and T cell responses. JVirol. 2017;91(9):e01596-16.

24. Durier C, et al. Clinical safety of HIV lipopeptides used as vaccines in healthy volunteers and HIVinfected adults. AIDS. 2006;20(7):1039-1049.

25. Gahéry-Ségard H, et al. Multiepitopic B-and $\mathrm{T}$-cell responses induced in humans by a human immunodeficiency virus type 1 lipopeptide vaccine. J Virol. 2000;74(4):1694-1703.

26. Pialoux G, et al. Lipopeptides induce cellmediated anti-HIV immune responses in seronegative volunteers. AIDS. 2001;15(10):1239-1249.

27. Lévy Y, et al. Immunological and virological efficacy of a therapeutic immunization combined 
with interleukin-2 in chronically HIV-1 infected patients. AIDS. 2005;19(3):279-286.

28. Gahery H, et al. New CD4+ and CD8+ T cell responses induced in chronically HIV type-1infected patients after immunizations with an HIV type 1 lipopeptide vaccine. AIDS Res Hum Retroviruses. 2006;22(7):684-694.

29. Klinguer C, et al. Characterization of a multilipopeptides mixture used as an HIV-1 vaccine candidate. Vaccine. 1999;18(3-4):259-267.

30. Lévy Y, et al. Sustained control of viremia following therapeutic immunization in chronically HIV1-infected individuals. AIDS. 2006;20(3):405-413.

31. Lévy Y, et al. Dendritic cell-based therapeutic vaccine elicits polyfunctional HIV-specific T-cell immunity associated with control of viral load. Eur J Immunol. 2014;44(9):2802-2810.

32. Grewal IS, Flavell RA. CD 40 and CD154 in cell-mediated immunity. Annu Rev Immunol. 1998;16:111-135.

33. Barr TA, McCormick AL, Carlring J, Heath AW. A potent adjuvant effect of CD40 antibody attached to antigen. Immunology. 2003;109(1):87-92.

34. Hangalapura BN, et al. Selective transduction of dendritic cells in human lymph nodes and superior induction of high-avidity melanoma-reactive cytotoxic $\mathrm{T}$ cells by a $\mathrm{CD} 40$-targeted adenovirus. JImmunother. 2010;33(7):706-715.

35. Schjetne KW, Fredriksen AB, Bogen B. Delivery of antigen to $\mathrm{CD} 40$ induces protective immune responses against tumors. JImmunol. 2007;178(7):4169-4176.

36. Chatterjee B, et al. Internalization and endosomal degradation of receptor-bound antigens regulate the efficiency of cross presentation by human dendritic cells. Blood. 2012;120(10):2011-2020.

37. Cohn L, et al. Antigen delivery to early endosomes eliminates the superiority of human blood BDCA3+ dendritic cells at cross presentation. JExp Med. 2013;210(5):1049-1063.

38. Yin $\mathrm{W}$, et al. Functional specialty of CD40 and dendritic cell surface lectins for exogenous antigen presentation to CD8(+) and CD4(+) T cells. EBioMedicine. 2016;5:46-58.

39. Flamar AL, et al. Targeting concatenated HIV antigens to human $\mathrm{CD} 40$ expands a broad repertoire of multifunctional CD4+ and CD8+ T cells. AIDS. 2013;27(13):2041-2051.

40. Cheng L, et al. Human innate responses and adjuvant activity of TLR ligands in vivo in mice reconstituted with a human immune system. Vaccine. 2017;35(45):6143-6153.

41. Shultz LD, Ishikawa F, Greiner DL. Humanized mice in translational biomedical research. Nat Rev Immunol. 2007;7(2):118-130.

42. Shultz LD, Brehm MA, Garcia-Martinez JV, Greiner DL. Humanized mice for immune system investigation: progress, promise and challenges. Nat Rev Immunol. 2012;12(11):786-798.

43. Lan P, Tonomura N, Shimizu A, Wang S, Yang YG. Reconstitution of a functional human immune system in immunodeficient mice through combined human fetal thymus/liver and CD34+ cell transplantation. Blood. 2006;108(2):487-492.

44. Melkus MW, et al. Humanized mice mount specific adaptive and innate immune responses to EBV and TSST-1. Nat Med. 2006;12(11):1316-1322.

45. Cheng L, et al. Blocking type I interferon signaling enhances $\mathrm{T}$ cell recovery and reduces HIV-1 reservoirs. J Clin Invest. 2017;127(1):269-279.

46. Cheng L, et al. Type I interferons suppress viral replication but contribute to $\mathrm{T}$ cell depletion and dysfunction during chronic HIV-1 infection. JCI Insight. 2017;2(12):94366.

47. Zhang L, Su L. HIV-1 immunopathogenesis in humanized mouse models. Cell Mol Immunol. 2012;9(3):237-244.

48. Marsden MD, Zack JA. Humanized mouse models for human immunodeficiency virus infection. Annu Rev Virol. 2017;4(1):393-412.

49. Bachem A, et al. Superior antigen crosspresentation and XCR1 expression define human CD11c+CD141+ cells as homologues of mouse CD8+ dendritic cells. JExp Med. 2010;207(6):1273-1281.

50. Crozat K, et al. The XC chemokine receptor 1 is a conserved selective marker of mammalian cells homologous to mouse CD8alpha+ dendritic cells. J Exp Med. 2010;207(6):1283-1292.

51. Jongbloed SL, et al. Human CD141+ (BDCA-3)+ dendritic cells (DCs) represent a unique myeloid DC subset that cross-presents necrotic cell antigens. JExp Med. 2010;207(6):1247-1260.

52. Poulin LF, et al. Characterization of human DNGR-1+ BDCA3+ leukocytes as putative equivalents of mouse CD8alpha+ dendritic cells. J Exp Med. 2010;207(6):1261-1271.

53. Archin NM, et al. Administration of vorinostat disrupts HIV-1 latency in patients on antiretroviral therapy. Nature. 2012;487(7408):482-485.

54. Deeks SG. HIV: Shock and kill. Nature. 2012;487(7408):439-440.

55. Novis CL, et al. Reactivation of latent HIV-1 in central memory $\mathrm{CD}^{+}{ }^{+} \mathrm{T}$ cells through TLR-1/2 stimulation. Retrovirology. 2013;10:119.

56. Alvarez-Carbonell D, et al. Toll-like receptor 3 activation selectively reverses HIV latency in microglial cells. Retrovirology. 2017;14(1):9.

57. Krarup AR, et al. The TLR9 agonist MGN1703 triggers a potent type I interferon response in the sigmoid colon. Mucosal Immunol. 2018;11(2):449-461.

58. Whitney JB, et al. Treatment with a TLR7 agonist induces transient viremia in SIV-infected ARTsuppressed monkeys. In: Conference on Retroviruses and Opportunistic Infections; February 23-26, 2015; Seattle, WA. Abstract 108.

59. Banga R, Procopio FA, Cavassini M, Perreau M. In vitro reactivation of replication-competent and infectious HIV-1 by histone deacetylase inhibitors. J Virol. 2016;90(4):1858-1871.

60. Coffman RL, Sher A, Seder RA. Vaccine adjuvants: putting innate immunity to work. Immunity. 2010;33(4):492-503

61. Walker-Sperling VE, Pohlmeyer CW, Tarwater PM, Blankson JN. The effect of latency reversal agents on primary CD8+ T cells: implications for shock and kill strategies for human immunodeficiency virus eradication. EBioMedicine. 2016;8:217-229.

62. Kelly-Sell MJ, et al. The histone deacetylase inhibitor, romidepsin, suppresses cellular immune functions of cutaneous T-cell lymphoma patients. Am J Hematol. 2012;87(4):354-360.

63. Fukazawa $Y$, et al. B cell follicle sanctuary permits persistent productive simian immunodeficiency virus infection in elite controllers. Nat Med. 2015;21(2):132-139.

64. Perreau M, et al. Follicular helper T cells serve as the major CD4 $\mathrm{T}$ cell compartment for HIV-1 infection, replication, and production. J Exp Med. 2013;210(1):143-156.

65. Namikawa R, Kaneshima H, Lieberman $\mathrm{M}$, Weissman IL, McCune JM. Infection of the SCID-hu mouse by HIV-1. Science. 1988;242(4886):1684-1686.

66. Halper-Stromberg A, et al. Broadly neutralizing antibodies and viral inducers decrease rebound from HIV-1 latent reservoirs in humanized mice. Cell. 2014;158(5):989-999. 